\title{
Global asymptotic stability beyond 3/2 type stability for a logistic equation with piecewise constant arguments
}

\author{
Yukihiko Nakata* \\ BCAM - Basque Center For Applied Mathematics, Bizkaia Technology Park, Building 500 E-48160 Derio, Spain \\ Department of Pure and Applied Mathematics, Waseda University, 3-4-1, Ohkubo, Shinjuku-ku, Tokyo, Japan
}

\section{A R T I C L E I N F O}

\section{Article history:}

Received 14 May 2009

Accepted 28 June 2010

\section{MSC:}

39A10

39A11

92D25

\section{Keywords:}

Global asymptotic stability

Difference equations

Piecewise constant arguments

Time delays

Logistic equation

$3 / 2$ type stability

\begin{abstract}
A B S T R A C T
In this paper, a logistic equation with multiple piecewise constant arguments is investigated in detail. We generalize the approach in two papers, [K. Uesugi, Y. Muroya, E. Ishiwata, On the global attractivity for a logistic equation with piecewise constant arguments, J. Math. Anal. Appl. 294 (2) (2004) 560-580] and [Y. Muroya, E. Ishiwata, N. Guglielmi, Global stability for nonlinear difference equations with variable coefficients, J. Math. Anal. Appl. 334 (1) (2007) 232-247], and establish a new condition for the global stability of the equation. Their results are given as one of the special cases. Moreover, we improve the $3 / 2$ type stability condition under several dominance assumptions on the coefficients of the equation. Some examples and numerical simulations are also presented. All of these examples show that there are several conditions for the global stability of the equation, depending on the coefficients on the delay terms of the equation, beyond the $3 / 2$ type stability condition.
\end{abstract}

(c) 2010 Elsevier Ltd. All rights reserved.

\section{Introduction}

In this paper, we consider the following logistic equation with piecewise constant arguments:

$$
\frac{\mathrm{d} N(t)}{\mathrm{d} t}=N(t) r\left(1-\sum_{j=0}^{m} b_{j} N([t-j])\right),
$$

where

$$
r>0, \quad b_{j} \geq 0, \quad j=0,1,2, \ldots, m, \quad \text { and } \quad \sum_{j=0}^{m} b_{j}>0,
$$

with initial condition $N(-l)=N_{-l} \geq 0, l=1,2, \ldots, m$ and $N(0)=N_{0}>0$. [t] means the maximal integer not greater than $t$. (1.1) has the positive equilibrium $N^{*}=1 /\left(\sum_{j=0}^{m} b_{j}\right)$.

Several classes of nonlinear delay differential and difference equations have been studied many times in the literature (see [1-20] and references therein) and many authors focus on the global stability of the equation as one of the important topics from the viewpoint of the application. Gopalsamy and Liu [1] studied a class of logistic equations with one piecewise constant argument and offered a conjecture about the global asymptotic stability of the equation. Recently, the

\footnotetext{
* Corresponding address: BCAM - Basque Center For Applied Mathematics, Bizkaia Technology Park, Building 500 E-48160 Derio, Spain. Tel.: +34 946 567853.

E-mail address: nakata@bcamath.org.
} 
conjecture has been completely solved by Muroya and Kato [13] and Li and Yuan [3]. A more general logistic equation with piecewise constant arguments was studied by Muroya [8] and the result was extended to the nonautonomous case by Nakata et al. [14].

The main purpose in the present paper is to investigate (1.1) in detail and establish a new sufficient condition for the global asymptotic stability. Several authors have investigated the stability and oscillatory characteristics of (1.1) with $m=0$, and it is known that $r \leq 2$ is the necessary and sufficient condition for the global attractivity of the positive equilibrium $N^{*}=1 / b_{0}$.

Theorem A (See [6, Theorem 1.1]). Let $m=0$. If

$$
r \leq 2 \text {, }
$$

then for any solution $N(t)$ of (1.1), $\lim _{t \rightarrow+\infty} N(t)=N^{*}$.

For the case $m \geq 1$, So and Yu [15] obtained the following 3/2 type criterion for the global attractivity of the positive equilibrium $N^{*}=1 /\left(\sum_{j=0}^{m} b_{j}\right)$.

Theorem B (See [15, Theorem 1.2]). Let $m \geq 1$. If

$$
r \leq \frac{3}{2(m+1)}
$$

then for any solution $N(t)$ of $(1.1), \lim _{t \rightarrow+\infty} N(t)=N^{*}$.

For the global asymptotic stability of the delay differential and difference equations, $3 / 2$ type stability conditions are obtained in many literature entries (see, for example, [7,16-18] and references therein). In particular, Theorem B holds for any set of the coefficients of the equation. On the other hand, Uesugi et al. [19] paid attention to the following dominance assumption:

$$
b_{0}>\sum_{j=1}^{m} b_{j} .
$$

They established a better condition for the global attractivity of (1.1) under (1.5).

Theorem C (See [19, Theorem 1.2]). Let $m \geq 1$. Suppose that (1.5) holds. If

$$
r \leq 2 \text {, }
$$

and

$$
r-\frac{\sum_{j=1}^{m} b_{j}}{b_{0}} \exp (r-1) \geq 0 \quad \text { for } r>1,
$$

then for any solution $N(t)$ of (1.1), $\lim _{t \rightarrow+\infty} N(t)=N^{*}$ and the positive equilibrium $N^{*}$ is globally asymptotically stable.

One can see that under the assumption (1.5), they really improve Theorem B. Moreover, by [19, Corollary 1.1], we see that (1.3) still guarantees the global asymptotic stability of (1.1) if $\sum_{j=1}^{m} b_{j} / b_{0} \leq 2 / e$.

Now, a natural question occurs, that is, whether or not the bound $3 / 2(m+1)$ in $(1.4)$ is the best possible constant for the global stability of (1.1), when (1.5) is not true. In this paper, using a generalized approach from [19,12], we offer affirmative answers for the above question.

Instead of (1.5), throughout the paper, we impose the following general assumption.

(H) There exists an integer $i \in[0, m]$ such that

$$
\sum_{k=0}^{i} \sum_{j=0}^{i-k} b_{j}>\sum_{k=0}^{i} \sum_{j=i-k+1}^{m} b_{j} .
$$

For a given set of the coefficients, $b_{j}, j=0,1,2, \ldots, m$, for (1.1), if we can have (H) with $i=0$, then (1.8) becomes (1.5). Therefore, $(\mathrm{H})$ is a generalized assumption from (1.5). Under the assumption $(\mathrm{H})$, we establish the following new condition for the global asymptotic stability of (1.1), which generalizes the results given in $[9,19,12]$.

Theorem 1.1. Let $m \geq 1$. Suppose that $(\mathrm{H})$ holds. If

$$
r \leq \frac{2}{i+1},
$$


and

$$
r(i+1)-\frac{\sum_{k=0}^{i} \sum_{j=i-k+1}^{m} b_{j}}{\sum_{k=0}^{i} \sum_{j=0}^{i-k} b_{j}} \exp (r(i+1)-1) \geq 0 \quad \text { for } r(i+1)>1,
$$

then for any solution $N(t)$ of $(1.1), \lim _{t \rightarrow+\infty} N(t)=N^{*}$ and the positive equilibrium $N^{*}$ is globally asymptotically stable. As a direct consequence, we have the following corollaries.

Corollary 1.2. Let $m \geq 1$. Assume that there exists an integer $i \in[0, m]$ such that

$$
\frac{\sum_{k=0}^{i} \sum_{j=i-k+1}^{m} b_{j}}{\sum_{k=0}^{i} \sum_{j=0}^{i-k} b_{j}} \leq \frac{2}{\mathrm{e}}
$$

If $r \leq \frac{2}{i+1}$, then for any solution $N(t)$ of $(1.1), \lim _{t \rightarrow+\infty} N(t)=N^{*}$ and the positive equilibrium $N^{*}$ is globally asymptotically stable.

Corollary 1.3. Let $m \geq 1$. Assume that there exists an integer $i \in[0, m]$ such that

$$
\frac{\sum_{k=0}^{i} \sum_{j=0}^{i-k} b_{j}}{(i+1) \sum_{j=0}^{m} b_{j}} \geq \frac{2}{e+2}
$$

If $r \leq \frac{2}{i+1}$, then for any solution $N(t)$ of (1.1), $\lim _{t \rightarrow+\infty} N(t)=N^{*}$ and the positive equilibrium $N^{*}$ is globally asymptotically stable.

For (1.1), if $b_{0}>\sum_{j=1}^{m} b_{j}$, then we can choose $i=0$, and Theorem 1.1 becomes Theorem $C$. For the case $i=m$, a similar result is established by Muroya et al. [12].

Moreover, we obtain the following several corollaries.

Corollary 1.4. Let $m \geq 1$. Suppose that $\sum_{k=0}^{1} \sum_{j=0}^{1-k} b_{j}>\sum_{k=0}^{1} \sum_{j=2-k}^{m} b_{j}$. If

$$
r \leq 1, \quad \text { and } \quad 2 r-\frac{\sum_{k=0}^{1} \sum_{j=2-k}^{m} b_{j}}{\sum_{k=0}^{1} \sum_{j=0}^{1-k} b_{j}} \exp (2 r-1) \geq 0 \text { for } r>\frac{1}{2},
$$

then for any solution $N(t)$ of $(1.1), \lim _{t \rightarrow+\infty} N(t)=N^{*}$ and the positive equilibrium $N^{*}$ is globally asymptotically stable.

Corollary 1.5. Let $m \geq 1$. Suppose that $\sum_{k=0}^{2} \sum_{j=0}^{2-k} b_{j}>\sum_{k=0}^{2} \sum_{j=3-k}^{m} b_{j}$. If

$$
r \leq \frac{2}{3}, \quad \text { and } \quad 3 r-\frac{\sum_{k=0}^{2} \sum_{j=3-k}^{m} b_{j}}{\sum_{k=0}^{2} \sum_{j=0}^{2-k} b_{j}} \exp (3 r-1) \geq 0 \text { for } r>\frac{1}{3},
$$

then for any solution $N(t)$ of $(1.1), \lim _{t \rightarrow+\infty} N(t)=N^{*}$ and the positive equilibrium $N^{*}$ is globally asymptotically stable.

To illustrate the above results, we introduce an example.

Example 1.6. Consider the following logistic equation with two piecewise constant arguments:

$$
\frac{\mathrm{d} N(t)}{\mathrm{d} t}=N(t) r(1-0.2 N([t])-0.8 N([t-1])) .
$$


(1.15) has the positive equilibrium $N^{*}=1$. We can choose $i=1$ such that $(\mathrm{H})$ holds and it follows that $0.8 / 1.2 \leq 2 / e$. By Corollary 1.2, if $0<r \leq 1$, then for any solution $N(t)$ of (1.15), $\lim _{t \rightarrow+\infty} N(t)=N^{*}=1$ and the positive equilibrium $N^{*}=1$ is globally asymptotically stable. On the other hand, (1.4) becomes $r \leq \frac{3}{4}=0.75$ and, hence, our stability condition improves Theorem B without the assumption (1.5).

This paper is organized as follows. In Section 2, we prepare some basic results and derive a new condition for the global asymptotic stability in Lemma 2.6. Lemma 2.6 is the most important result in this paper and established by a generalized approach of the estimation of the upper and lower bounds of the solution from Uesugi et al. [19] and Muroya et al. [12]. In the proof of Lemma 2.6, Lemma 2.5 becomes one of the key results. In Section 3, using the property of a unimodal function $\varphi(x)$, which is introduced in Section 2, some sufficient conditions for Lemma 2.6 are established. Moreover, we introduce Theorem 3.6, which extends the previous results in $[9,20]$. Using the previous results given in [19], we finally prove Theorem 1.1 in the last part of Section 3. In Section 4, some examples and numerical simulations are given. All of these examples show that there are several conditions for the global stability of the equation, depending on the coefficients on the delay terms of the equation, beyond the $3 / 2$ type stability condition.

\section{Basic results}

In this section, we prepare some basic results. With a new approach of the estimation of the upper and lower bounds of the solution, we establish a condition for the global asymptotic stability of the equation in Lemma 2.6.

At first, under a variable transformation $N(t)=N^{*} \exp (x(t)),(1.1)$ becomes

$$
\frac{\mathrm{d} x(t)}{\mathrm{d} t}=-\sum_{j=0}^{m} a_{j} f(x([t-j])),
$$

where

$$
\left\{\begin{array}{l}
a_{j}=r N^{*} b_{j} \text { for } j=0,1,2, \ldots, m, \\
f(x)=\mathrm{e}^{x}-1
\end{array}\right.
$$

with initial condition $x(-l)=\ln \left(N_{-l} / N^{*}\right), l=0,1,2, \ldots, m$. By integrating both sides of (2.1) from $n$ to $t$ on the interval $[n, n+1), n=0,1,2, \ldots$, we obtain that

$$
x(t)-x(n)=-\sum_{j=0}^{m} a_{j} f(x(n-j))(t-n) .
$$

By the continuity of the solution, we let $t \rightarrow n+1$ and obtain the following discretized equation with delays:

$$
x(n+1)=x(n)-\sum_{j=0}^{m} a_{j} f(x(n-j)) .
$$

It is clear that (2.3) has the zero solution. The global asymptotic stability of the zero solution of (2.3) is equivalent to the global asymptotic stability of the positive equilibrium $N^{*}$ of (1.1). Hereafter, we consider (2.3).

Let us introduce the following well known results from Muroya et al. [12] (see also [15,19]).

Lemma 2.1 (See [12, Lemma 2.1]). Let $\{x(n)\}_{n=0}^{\infty}$ be the solution of (2.3). If $x(n)$ is eventually larger (resp. smaller) than 0 , then $x(n)$ is eventually decreasing (resp. increasing), and $\lim _{n \rightarrow \infty} x(n)$ exists and it holds that $\lim _{n \rightarrow \infty} x(n)=0$.

Next, we show the uniform persistence for (2.3).

Lemma 2.2 (See also [12, Lemma 2.2]). Let $\{x(n)\}_{n=0}^{\infty}$ be the solution of (2.3). If $x(n)$ is oscillatory about 0 , and $\sum_{j=0}^{m} a_{j}<+\infty$, then $x(n)$ is bounded above and below. Moreover, it holds that

$$
-\infty<-(m+1) \sum_{j=0}^{m} a_{j} f(M) \leq \liminf _{n \rightarrow+\infty} x(n) \leq \limsup _{n \rightarrow+\infty} x(n) \leq M<+\infty,
$$

where $M=(m+1) \sum_{j=0}^{m} a_{j}$.

Proof. From (2.2)-(2.3), we have that

$$
x(n+1)-x(n) \leq \sum_{j=0}^{m} a_{j}, \quad \text { for } n \geq 0 .
$$


Suppose that $\lim \sup _{n \rightarrow+\infty} x(n)=+\infty$. Then, there exists a strictly monotone increasing integer sequence $\left\{\bar{n}_{k}\right\}_{k=1}^{+\infty}$ such that $\bar{n}_{k} \geq 0$ and

$$
x\left(\bar{n}_{k}\right)=\max _{0 \leq n \leq \bar{n}_{k}} x(n), \quad x\left(\bar{n}_{k}\right) \geq x\left(\bar{n}_{k}-1\right) \quad \text { and } \quad \lim _{k \rightarrow+\infty} x\left(\bar{n}_{k}\right)=\limsup _{n \rightarrow+\infty} x(n) .
$$

Then, by (2.3) we have

$$
0 \leq x\left(\bar{n}_{k}\right)-x\left(\bar{n}_{k}-1\right)=-\sum_{j=0}^{m} a_{j} f\left(x\left(\bar{n}_{k}-1-j\right)\right) \quad \text { for } \bar{n}_{k}>m,
$$

which implies $0 \leq-\sum_{j=0}^{m} a_{j} f\left(x\left(\bar{n}_{k}-1-j\right)\right)$. Thus, there exists an integer $\bar{\eta}_{k} \in\left[\bar{n}_{k}-1-m, \bar{n}_{k}-1\right]$ such that $x\left(\bar{\eta}_{k}\right) \leq 0$. Summing (2.4) from $n=\bar{\eta}_{k}$ to $n=\bar{n}_{k}-1$, we have

$$
x\left(\bar{n}_{k}\right) \leq x\left(\bar{\eta}_{k}\right)+\left(\bar{n}_{k}-\bar{\eta}_{k}\right) \sum_{j=0}^{m} a_{j} \leq(m+1) \sum_{j=0}^{m} a_{j}=M .
$$

This leads to a contradiction to our assumption. Thus, $x(n)<+\infty$ for $n>0$.

Moreover, like in the above discussion, we obtain that $x(n) \leq M<+\infty$ for $n>0$ and $\lim _{\sup _{n \rightarrow+\infty}} x(n) \leq M<+\infty$.

Next, let us consider the lower bound of the solution. From (2.2)-(2.3), we have that

$$
x(n+1)-x(n) \geq-\sum_{j=0}^{m} a_{j} f(M), \quad \text { for } n \geq m+1 .
$$

Suppose that $\lim _{\inf } n_{n \rightarrow+\infty} x(n)=-\infty$. Then, there exists a strictly monotone increasing integer sequence $\left\{\underline{n}_{k}\right\}_{k=1}^{+\infty}$ such that $\underline{n}_{k} \geq 0$ and

$$
x\left(\underline{n}_{k}\right)=\min _{0 \leq n \leq \underline{n}_{k}} x(n), \quad x\left(\underline{n}_{k}\right) \leq x\left(\underline{n}_{k}-1\right) \quad \text { and } \quad \lim _{k \rightarrow+\infty} x\left(\underline{n}_{k}\right)=\liminf _{n \rightarrow+\infty} x(n) .
$$

Then, by (2.3) we have

$$
0 \geq x\left(\underline{n}_{k}\right)-x\left(\underline{n}_{k}-1\right)=-\sum_{j=0}^{m} a_{j} f\left(x\left(\underline{n}_{k}-1-j\right)\right) \quad \text { for } \underline{n}_{k}>2 m+1,
$$

which implies $0 \geq-\sum_{j=0}^{m} a_{j} f\left(x\left(\underline{n}_{k}-1-j\right)\right)$. Thus, there exists an integer $\underline{\eta}_{k} \in\left[\underline{n}_{k}-1-m, \underline{n}_{k}-1\right]$ such that $x\left(\underline{\eta}_{k}\right) \geq 0$. Summing (2.5) from $n=\underline{\eta}_{k}$ to $n=\underline{n}_{k}-1$, we have

$$
x\left(\underline{n}_{k}\right) \geq x\left(\underline{\eta}_{k}\right)+\left(\underline{n}_{k}-\underline{\eta}_{k}\right) \sum_{j=0}^{m} a_{j} f(M) \geq-(m+1) \sum_{j=0}^{m} a_{j} f(M) .
$$

This leads to a contradiction to our assumption. Thus, $x(n)>-\infty$ for $n>0$.

Moreover, like in the above discussion, we obtain that $x(n) \geq-(m+1) \sum_{j=0}^{m} a_{j} f(M)>-\infty$ for $n>2 m+1$ and $\liminf _{n \rightarrow+\infty} x(n) \geq-(m+1) \sum_{j=0}^{m} a_{j} f(M)>-\infty$. Hence, the proof is complete.

Remark 2.3. By Lemma 2.2, for some real number (small enough) $L<0$, there exists a positive integer $n_{L}$ such that $x(n) \geq L$ for $n \geq n_{L}$.

Under the assumption $(\mathrm{H})$, without loss of generality, we can fix $i \in[0, m]$ and define

$$
r_{1}=\sum_{k=0}^{i} \sum_{j=0}^{i-k} a_{j} \text { and } r_{2}=\sum_{k=0}^{i} \sum_{j=i-k+1}^{m} a_{j},
$$

respectively for such $i$. We note that $r_{1}+r_{2}=(i+1) \sum_{j=0}^{m} a_{j}$, and by (2.2) it holds that

$$
r_{1}+r_{2}=r(i+1) .
$$

Moreover, by $(\mathrm{H})$, throughout the paper, we assume that

$$
r_{1}>r_{2} \geq 0 \text {. }
$$

The following lemma is used in Lemma 2.6 and some discussions in Section 3. 
Lemma 2.4. Let $\{x(n)\}_{n=0}^{\infty}$ be the solution of (2.3). Suppose that for some real number $L<0$, there exists a positive integer $n_{L}$ such that $x(n) \geq L$ for $n \geq n_{L}$. If there exists an integer $n>n_{L}+2 m$ such that $x(n+1)>0$ and $x(n+1) \geq x(n)$, then there exists an integer $\underline{g}(n) \in[0, i]$ such that

$$
x(n-\underline{g}(n))=\min _{0 \leq j \leq i} x(n-j) \leq f^{-1}\left(-\frac{\sum_{j=i+1}^{m} a_{j}}{\sum_{j=0}^{i} a_{j}} f(L)\right) .
$$

Moreover, it holds that

$$
\frac{\sum_{j=i+1}^{m} a_{j}}{\sum_{j=0}^{i} a_{j}} \leq \frac{\sum_{k=0}^{1} \sum_{j=i-k+1}^{m} a_{j}}{\sum_{k=0}^{1} \sum_{j=0}^{i-k} a_{j}} \leq \frac{\sum_{k=0}^{2} \sum_{j=i-k+1}^{m} a_{j}}{\sum_{k=0}^{2} \sum_{j=0}^{i-k} a_{j}} \leq \cdots \leq \frac{r_{2}}{r_{1}},
$$

and it follows that

$$
x(n-\underline{g}(n)) \leq f^{-1}\left(-\frac{\sum_{j=i+1}^{m} a_{j}}{\sum_{j=0}^{i} a_{j}} f(L)\right) \leq f^{-1}\left(-\frac{r_{2}}{r_{1}} f(L)\right) .
$$

Proof. From (2.6) and (2.8), it is clear that $\sum_{j=0}^{i} a_{j}>0$, if $r_{1}>0$. From (2.3), we have that

$$
0 \leq x(n+1)-x(n) \leq-\sum_{j=0}^{i} a_{j} f(x(n-\underline{g}(n)))-\sum_{j=i+1}^{m} a_{j} f(L),
$$

which implies

$$
f(x(n-\underline{g}(n))) \leq-\frac{\sum_{j=i+1}^{m} a_{j}}{\sum_{j=0}^{i} a_{j}} f(L),
$$

and (2.9) holds. From the observation of the following inequality:

$$
\frac{y_{2}}{y_{1}} \leq \frac{y_{2}+\tilde{y_{2}}}{y_{1}+\tilde{y_{1}}} \text { for } 0 \leq y_{2} \leq \tilde{y_{2}} \text { and } 0 \leq \tilde{y_{1}} \leq y_{1},
$$

we see that (2.10) holds. By (2.9) and (2.10), we easily obtain (2.11). Hence, the proof is complete.

The following observation enables us to establish Lemma 2.6.

Lemma 2.5. Let $\{x(n)\}_{n=0}^{\infty}$ be the solution of (2.3). Suppose that for some real number $L<0$, there exists a positive integer $n_{L}$ such that $x(n) \geq L$ for $n \geq n_{L}$. If there exists an integer $n>n_{L}+2 m$ such that $x(n+1)>0$ and $x(n+1) \geq x\left(n-\left(i-i_{1}\right)\right)$ for an integer $i_{1} \in[0, i]$, then there exists an integer $\underline{g}(n) \in[0, i]$ such that

$$
x(n-\underline{g}(n))=\min _{0 \leq j \leq i} x(n-j),
$$

and it holds that

$$
-\left(\sum_{k=i_{1}}^{i} \sum_{j=0}^{i-k} a_{j}\right) f(x(n-\underline{g}(n)))-\left(\sum_{k=i_{1}}^{i} \sum_{j=i-k+1}^{m} a_{j}\right) f(L) \geq 0 .
$$

Moreover, suppose that for some real number $R_{L}>0$, it holds that $x(n) \leq R_{L}$ for $n>n_{L}+4 m+1$. If there exists an integer $n>n_{L}+4 m+1$ such that $x(n+1)<0$ and $x(n+1) \leq x\left(n-\left(i-i_{2}\right)\right)$ for an integer $i_{2} \in[0$, $i]$, then there exists an integer $\bar{g}(n) \in[0, i]$ such that

$$
x(n-\bar{g}(n))=\max _{0 \leq j \leq i} x(n-j),
$$


and it holds that

$$
-\left(\sum_{k=i_{2}}^{i} \sum_{j=0}^{i-k} a_{j}\right) f(x(n-\bar{g}(n)))-\left(\sum_{k=i_{2}}^{i} \sum_{j=i-k+1}^{m} a_{j}\right) f\left(R_{L}\right) \leq 0 .
$$

Proof. Since $(n-\underline{g}(n))-m \geq n-2 m$, we exploit (2.3) and get the following equations:

$$
\left\{\begin{array}{l}
x(n+1) \leq x(n)-\sum_{j=0}^{i-i_{1}} a_{j} f(x(n-\underline{g}(n)))-\sum_{j=i-i_{1}+1}^{m} a_{j} f(L), \\
x(n) \leq x(n-1)-\sum_{j=0}^{i-i_{1}-1} a_{j} f(x(n-\underline{g}(n)))-\sum_{j=i-i_{1}}^{m} a_{j} f(L), \\
x(n-1) \leq x(n-2)-\sum_{j=0}^{i-i_{1}-2} a_{j} f(x(n-\underline{g}(n)))-\sum_{j=i-i_{1}-1}^{m} a_{j} f(L), \\
\ldots \\
x\left(n-\left(i-i_{1}\right)+1\right) \leq x\left(n-\left(i-i_{1}\right)\right)-a_{0} f(x(n-\underline{g}(n)))-\sum_{j=1}^{m} a_{j} f(L),
\end{array}\right.
$$

and, hence, it follows that

$$
0 \leq x(n+1)-x\left(n-\left(i-i_{1}\right)\right) \leq-\left(\sum_{k=i_{1}}^{i} \sum_{j=0}^{i-k} a_{j}\right) f(x(n-\underline{g}(n)))-\left(\sum_{k=i_{1}}^{i} \sum_{j=i-k+1}^{m} a_{j}\right) f(L) .
$$

Thus, (2.12) holds. Similarly, (2.13) also holds and the proof is complete.

Before introducing the next lemma, we put

$$
d_{L}=f^{-1}\left(-\frac{r_{2}}{r_{1}} f(L)\right) \text { for } L<0
$$

and

$$
\varphi(x)=x-r_{1} f(x)
$$

We generalize the approach in $[19,12]$ and establish the following important lemma in this paper.

Lemma 2.6. Suppose that the solution $x(n)$ of (2.3) is oscillatory about 0 . If, for some real number $L<0$, there exists a positive integer $n_{L}$ such that $x(n) \geq L$ for $n \geq n_{L}$, then it holds that

$$
x(n+1) \leq R_{L}, n>n_{L}+2 m \text { and } x(n+1) \geq S_{L}, n>n_{L}+4 m+1,
$$

where

$$
R_{L}=\max _{L \leq x \leq d_{L}} \varphi(x)-r_{2} f(L) \quad \text { and } \quad S_{L}=\min _{L \leq x \leq R_{L}} \varphi(x)-r_{2} f\left(R_{L}\right),
$$

respectively. Moreover, if

$$
S_{L}>L \text { for any } L<0,
$$

then it holds that $\lim _{n \rightarrow+\infty} x(n)=0$.

Proof. Assume $x(n) \geq L$ for any $n \geq n_{L}$. From the assumption that the solution is oscillatory about 0 and Lemma 2.2 , there exists a strictly monotone increasing integer subsequence $\left\{\bar{n}_{k}\right\}_{k=1}^{+\infty}$ such that $\bar{n}_{k}>n_{L}+2 m$, and

$$
x\left(\bar{n}_{k}\right)=\max _{n_{L}+2 m<n<\bar{n}_{k}} x(n), \quad x\left(\bar{n}_{k}\right) \geq x\left(\bar{n}_{k}-1\right) \quad \text { and } \lim _{k \rightarrow+\infty} x\left(\bar{n}_{k}\right)=\limsup _{n \rightarrow+\infty} x(n) .
$$

Moreover, there exists an integer $\underline{g}(n) \in[0, i]$ for $n>n_{L}+2 m$ such that $x(n-\underline{g}(n))=\min _{0 \leq j \leq i} x(n-j)$ and, hence, we have $x(n-\underline{g}(n)) \leq x(n-j)$ for $j \stackrel{\underline{g}}{=} 0,1,2, \ldots, i$. For simplicity, we put

$$
x\left(\underline{\xi}_{k}\right)=x\left(\bar{n}_{k}-1-\underline{g}\left(\bar{n}_{k}-1\right)\right) .
$$


Since $(n-g(n))-m \geq n-2 m$, we exploit (2.3) and get the following equations:

$$
\left\{\begin{array}{l}
x\left(\bar{n}_{k}\right) \leq x\left(\bar{n}_{k}-1\right)-\sum_{j=0}^{i} a_{j} f\left(x\left(\underline{\xi}_{k}\right)\right)-\sum_{j=i+1}^{m} a_{j} f(L), \\
x\left(\bar{n}_{k}-1\right) \leq x\left(\bar{n}_{k}-2\right)-\sum_{j=0}^{i-1} a_{j} f\left(x\left(\underline{\xi}_{k}\right)\right)-\sum_{j=i}^{m} a_{j} f(L), \\
x\left(\bar{n}_{k}-2\right) \leq x\left(\bar{n}_{k}-3\right)-\sum_{j=0}^{i-2} a_{j} f\left(x\left(\underline{\xi}_{k}\right)\right)-\sum_{j=i-1}^{m} a_{j} f(L), \\
\ldots \\
x\left(\underline{\xi}_{k}+1\right) \leq x\left(\underline{\xi}_{k}\right)-\sum_{j=0}^{i-g\left(\bar{n}_{k}-1\right)} a_{j} f(x(\underline{\xi}))-\sum_{j=i-\underline{g}\left(\bar{n}_{k}-1\right)+1}^{m} a_{j} f(L),
\end{array}\right.
$$

and then we obtain that

$$
x\left(\bar{n}_{k}\right) \leq x\left(\underline{\xi}_{k}\right)-\left(\sum_{k=0}^{\underline{g}\left(\bar{n}_{k}-1\right)} \sum_{j=0}^{i-k} a_{j}\right) f\left(x\left(\underline{\xi}_{k}\right)\right)-\left(\sum_{k=0}^{\underline{g}\left(\bar{n}_{k}-1\right)} \sum_{j=i-k+1}^{m} a_{j}\right) f(L) .
$$

If $\underline{g}\left(\bar{n}_{k}-1\right) \leq i-1$, then we put $i_{1}=\underline{g}\left(\bar{n}_{k}-1\right)+1$ and, by Lemma 2.5 , we have that

$$
0 \leq-\left(\sum_{k=\underline{g}\left(\bar{n}_{k}-1\right)+1}^{i} \sum_{j=0}^{i-k} a_{j}\right) f\left(x\left(\underline{\xi}_{k}\right)\right)-\left(\sum_{k=\underline{g}\left(\bar{n}_{k}-1\right)+1}^{i} \sum_{j=i-k+1}^{m} a_{j}\right) f(L) .
$$

By adding (2.19) and (2.20), we obtain that

$$
x\left(\bar{n}_{k}\right) \leq x\left(\underline{\xi}_{k}\right)-\left(\sum_{k=0}^{i} \sum_{j=0}^{i-k} a_{j}\right) f\left(x\left(\underline{\xi}_{k}\right)\right)-\left(\sum_{k=0}^{i} \sum_{j=i-k+1}^{m} a_{j}\right) f(L)=\varphi\left(x\left(\underline{\xi}_{k}\right)\right)-r_{2} f(L) .
$$

From (2.11) in Lemma 2.4 and (2.14), we have $L \leq x\left(\xi_{k}\right) \leq d_{L}$. Thus, we obtain that $x(n+1) \leq R_{L}$ for any $n>n_{L}+2 m$. For the case $g\left(\bar{n}_{k}-1\right)=i$, we also obtain $x(n+1) \leq R_{L}$ for any $n>n_{L}+2 m$.

Like in the above discussion, there exists a strictly monotone increasing integer subsequence $\left\{\underline{n}_{k}\right\}_{k=1}^{+\infty}$ such that $\underline{n}_{k}>$ $n_{L}+4 m+1$, and

$$
x\left(\underline{n}_{k}\right)=\min _{n_{L}+4 m+1<n \leq \underline{n}_{k}} x(n), \quad x\left(\underline{n}_{k}\right) \leq x\left(\underline{n}_{k}-1\right) \quad \text { and } \quad \lim _{k \rightarrow+\infty} x\left(\underline{n}_{k}\right)=\liminf _{n \rightarrow+\infty} x(n) .
$$

There exists an integer $\bar{g}(n) \in[0, i]$ for $n>n_{L}+4 m+1$ such that $x(n-\bar{g}(n))=\max _{0 \leq j \leq i} x(n-j)$ and, hence, we have $x(n-\bar{g}(n)) \geq x(n-j)$ for $j=0,1,2, \ldots, i$. For simplicity, we put

$$
x\left(\bar{\xi}_{k}\right)=x\left(\underline{n}_{k}-1-\bar{g}\left(\underline{n}_{k}-1\right)\right) \text {. }
$$

Since $(n-g(n))-m \geq n-2 m$, we exploit (2.3) and get the following equations:

$$
\left\{\begin{array}{l}
x\left(\underline{n}_{k}\right) \geq x\left(\underline{n}_{k}-1\right)-\sum_{j=0}^{i} a_{j} f\left(x\left(\bar{\xi}_{k}\right)\right)-\sum_{j=i+1}^{m} a_{j} f\left(R_{L}\right) \\
x\left(\underline{n}_{k}-1\right) \geq x\left(\underline{n}_{k}-2\right)-\sum_{j=0}^{i-1} a_{j} f\left(x\left(\bar{\xi}_{k}\right)\right)-\sum_{j=i}^{m} a_{j} f\left(R_{L}\right) \\
x\left(\underline{n}_{k}-2\right) \geq x\left(\underline{n}_{k}-3\right)-\sum_{j=0}^{i-2} a_{j} f\left(x\left(\bar{\xi}_{k}\right)\right)-\sum_{j=i-1}^{m} a_{j} f\left(R_{L}\right) \\
\ldots \\
x\left(\bar{\xi}_{k}+1\right) \geq x\left(\bar{\xi}_{k}\right)-\sum_{j=0}^{i-\bar{g}\left(\underline{n}_{k}-1\right)} a_{j} f\left(x\left(\bar{\xi}_{k}\right)\right)-\sum_{j=i-\bar{g}\left(\underline{n}_{k}-1\right)+1}^{m} a_{j} f\left(R_{L}\right),
\end{array}\right.
$$

and then, we obtain that

$$
x\left(\underline{n}_{k}\right) \geq x\left(\bar{\xi}_{k}\right)-\left(\sum_{k=0}^{\bar{g}\left(\underline{n}_{k}-1\right)} \sum_{j=0}^{i-k} a_{j}\right) f\left(x\left(\bar{\xi}_{k}\right)\right)-\left(\sum_{k=0}^{\bar{g}\left(\underline{n}_{k}-1\right)} \sum_{j=i-k+1}^{m} a_{j}\right) f\left(R_{L}\right) .
$$


If $\bar{g}\left(\underline{n}_{k}-1\right) \leq i-1$, then we put $i_{2}=\underline{g}\left(\bar{n}_{k}-1\right)+1$ and by Lemma 2.5 , it holds that

$$
0 \geq-\left(\sum_{k=\bar{g}\left(\underline{n}_{k}-1\right)+1}^{i} \sum_{j=0}^{i-k} a_{j}\right) f\left(x\left(\bar{\xi}_{k}\right)\right)-\left(\sum_{k=\bar{g}\left(\underline{n}_{k}-1\right)+1}^{i} \sum_{j=i-k+1}^{m} a_{j}\right) f\left(R_{L}\right) .
$$

Consequently, by adding (2.21) and (2.22), we obtain that

$$
x\left(\underline{n}_{k}\right) \geq x\left(\bar{\xi}_{k}\right)-\left(\sum_{k=0}^{i} \sum_{j=0}^{i-k} a_{j}\right) f\left(x\left(\bar{\xi}_{k}\right)\right)-\left(\sum_{k=0}^{i} \sum_{j=i-k+1}^{m} a_{j}\right) f\left(R_{L}\right)=\varphi\left(x\left(\bar{\xi}_{k}\right)\right)-r_{2} f\left(R_{L}\right) .
$$

Now we have $L \leq x\left(\bar{\xi}_{k}\right) \leq R_{L}$. Hence, we obtain that $x(n+1) \geq S_{L}$ for any $n>n_{L}+4 m+1$. For the case $\bar{g}\left(\underline{n}_{k}-1\right)=i$, we easily obtain the same conclusion.

Next, we suppose that $S_{L}>L$ for any $L<0$. We set $\underline{L}=\liminf _{n \rightarrow+\infty} x(n)$ and if $\underline{L}<0$, then we have that $S_{\underline{L}}>\underline{L}$ for such a $\underline{L}<0$. Therefore, there exists an integer $\bar{n}_{\underline{L}}$ such that

$$
x(n) \geq S_{\underline{L}}>\underline{L}, \quad \text { for any } n>\bar{n}_{\underline{L}},
$$

which is a contradiction to the assumption, that is, $\underline{L}=\liminf _{n \rightarrow+\infty} x(n)$. Hence, we have $\underline{L}=0$ and this implies that $\lim _{n \rightarrow+\infty} x(n)=0$. Hence, the proof is complete.

Remark 2.7. By Lemmas $2.1,2.2$ and 2.6 , the zero solution of (2.3) is uniformly asymptotically stable. Hence, $\lim _{n \rightarrow+\infty} x(n)=0$ implies that the zero solution of (2.3) is globally asymptotically stable.

Remark 2.8. Muroya et al. [12] and So and Yu [15] pay attention to the fact that there exists at least one nonpositive delay term when they estimate the upper bound of the solution. However, our approach does not need such a observation. A similar statement is true for the estimation of the lower bound of the solution.

\section{Explicit conditions for Lemma 2.6 and the proof of Theorem 1.1}

In this section, we offer some conditions which ensure that (2.18) in Lemma 2.6 holds. Using the property of the unimodal function $\varphi(x)$, Theorems 3.1-3.3 are derived. Next, we establish one of the main theorems, which extends the results given in $[9,20]$. Moreover, we introduce useful results from Uesugi et al. [19] and finally prove Theorem 1.1.

The following important results are derived for Lemma 2.6.

Theorem 3.1. Assume that $\varphi(x)$ has only one critical point $L^{*}<0$ which is a local maximum point. If it holds that

$$
\min \left\{\varphi(L), \varphi\left(R_{L}\right)\right\}-r_{2} f\left(R_{L}\right)>L \text { for any } L<0,
$$

where

$$
R_{L}=\varphi\left(\max \left\{L^{*}, L\right\}\right)-r_{2} f(L),
$$

then the zero solution of (2.3) is globally asymptotically stable.

Proof. Now, $\varphi(x)$ is a unimodal function which attains a local maximum at $L^{*}<0$; hence, it follows that $\max _{L \leq x \leq d_{L}} \varphi(x)=$ $\varphi\left(\max \left\{L^{*}, L\right\}\right)$ and $R_{L}=\varphi\left(\max \left\{L^{*}, L\right\}\right)-r_{2} f(L)$ for $L<0$. It also holds that $\min \varphi_{L \leq x \leq R_{L}}(x)=\min \left\{\varphi(L), \varphi\left(R_{L}\right)\right\}$ and $S_{L}=\min \left\{\varphi(L), \varphi\left(R_{L}\right)\right\}-r_{2} f\left(R_{L}\right)$ for $L<0$. Hence, (3.1) implies that (2.18) holds. By Lemma 2.6, we have the conclusion of this theorem.

Theorem 3.2. Assume that $\varphi(x)$ has only one critical point $R^{*}>0$ which is a local maximum point.

(1) If $R^{*} \geq \varphi\left(R^{*}\right)+r_{2}$ and

$$
\varphi(L)-r_{2} f\left(R_{L}\right)>L \text { for any } L<0,
$$

where

$$
R_{L}=\varphi\left(d_{L}\right)-r_{2} f(L)=d_{L}
$$

then the zero solution of (2.3) is globally asymptotically stable.

(2) If $R^{*}<\varphi\left(R^{*}\right)+r_{2}$ then there exists a unique $\bar{L}<0$ such that $R^{*}=\varphi\left(R^{*}\right)-r_{2} f(\bar{L})$, and it holds that

$$
\left\{\begin{array}{l}
R^{*}>\varphi\left(R^{*}\right)-r_{2} f(L) \text { for } \bar{L}<L<0 \\
R^{*} \leq \varphi\left(R^{*}\right)-r_{2} f(L) \text { for } L \leq \bar{L}<0
\end{array}\right.
$$

Moreover, if

$$
\varphi(L)-r_{2} f\left(R_{L}\right)>L \text { for any } \bar{L}<L<0,
$$


where

$$
R_{L}=\varphi\left(d_{L}\right)-r_{2} f(L)=d_{L},
$$

and if

$$
\min \left\{\varphi(L), \varphi\left(R_{L}\right)\right\}-r_{2} f\left(R_{L}\right)>L \quad \text { for any } L \leq \bar{L}<0,
$$

where

$$
R_{L}=\varphi\left(R^{*}\right)-r_{2} f(L),
$$

then the zero solution of (2.3) is globally asymptotically stable.

Proof. (1) From the assumption that $R^{*} \geq \varphi\left(R^{*}\right)+r_{2}$, we see that $0 \geq-r_{1} f\left(R^{*}\right)+r_{2}$. Then, it follows that

$$
f\left(R^{*}\right) \geq \frac{r_{2}}{r_{1}} \geq-\frac{r_{2}}{r_{1}} f(L)=f\left(d_{L}\right) \text { for } L<0 .
$$

This implies that $d_{L} \leq R^{*}$ and, hence, it holds that $\max _{L \leq x \leq d_{L}} \varphi(x)=\varphi\left(d_{L}\right)$ and

$$
R_{L}=\varphi\left(d_{L}\right)-r_{2} f(L)=d_{L}-r_{1} f\left(d_{L}\right)-r_{2} f(L)=d_{L} \leq R^{*} \text { for } L<0,
$$

from which, we see that $\min _{L \leq x \leq R_{L}} \varphi(x)=\varphi(L)$. Hence, (3.2) implies that (2.18) holds. By Lemma 2.6, we have the conclusion.

(2) By [19, Lemma 2.4], there exists a unique $\bar{L}<0$ such that $R^{*}=\varphi\left(R^{*}\right)-r_{2} f(\bar{L})$. This implies that $0=-r_{1} f\left(R^{*}\right)-r_{2} f(\bar{L})$. Let us consider the case $\bar{L}<L<0$. From (2.14), it holds that

$$
d_{L}<f^{-1}\left(-\frac{r_{2}}{r_{1}} f(\bar{L})\right)=f^{-1}\left(f\left(R^{*}\right)\right)=R^{*} \text { for } \bar{L}<L<0,
$$

from which, we see that $\max _{L \leq x \leq d_{L}} \varphi(x)=\varphi\left(d_{L}\right)$ and $R_{L}=\varphi\left(d_{L}\right)-r_{2} f(L)=d_{L}<R^{*}$ for $\bar{L}<L<0$, and then it holds that $\min _{L \leq x \leq R_{L}} \varphi(x)=\varphi(L)$. Consequently, (3.3) implies that (2.18) holds. By Lemma 2.6, we have the conclusion.

Next, we consider the case $L \leq \bar{L}<0$. In this case, we have that $R^{*} \leq \varphi\left(R^{*}\right)-r_{2} f(L)$ for $L \leq \bar{L}<0$. Then, $0 \leq-r_{1} f\left(R^{*}\right)-r_{2} f(L)$ for $L \leq \bar{L}<\overline{0}$ and it follows that

$$
f\left(R^{*}\right) \leq-\frac{r_{2}}{r_{1}} f(L)=f\left(d_{L}\right) \quad \text { for } L \leq \bar{L}<0 .
$$

This implies that $0<R^{*} \leq d_{L}$ and, hence, we have that $\max _{L \leq x \leq d_{L}} \varphi(x)=\varphi\left(R^{*}\right)$ and

$$
R_{L}=\varphi\left(R^{*}\right)-r_{2} f(L) \geq R^{*} \text { for } L \leq \bar{L}<0 .
$$

Then, it follows that $\min _{L \leq x \leq R_{L}} \varphi(x)=\min \left\{\varphi(L), \varphi\left(R_{L}\right)\right\}$ and $S_{L}=\min \left\{\varphi(L), \varphi\left(R_{L}\right)\right\}-r_{2} f\left(R_{L}\right)$. Hence, (3.4) implies that (2.18) holds. By Lemma 2.6, we have the conclusion of this theorem.

Theorem 3.3. Assume that $\varphi(x)$ has only one critical point $R^{*}=0$ which is a local maximum point. Then $R^{*} \leq \varphi\left(R^{*}\right)+r_{2}$ and there exists a unique $\bar{L}=0$ such that $R^{*}=\varphi\left(R^{*}\right)-r_{2} f(\bar{L})$.

If it holds that

$$
\min \left\{\varphi(L), \varphi\left(R_{L}\right)\right\}-r_{2} f\left(R_{L}\right)>L \quad \text { for any } L<0,
$$

where

$$
R_{L}=\varphi\left(R^{*}\right)-r_{2} f(L),
$$

and then the zero solution of (2.3) is globally asymptotically stable.

Since the proof of Theorem 3.3 is similar to the proof of Theorem 3.2, we omit it here. For the convenience of the reader, we illustrate the graphs of $\varphi(x)$ in Figs. 1 and 2.

We put

$$
\tilde{\varphi}(x)=x-\left(r_{1}+r_{2}\right) f(x)
$$

and introduce the following useful results from Uesugi et al. [19] in Lemmas 3.4, 3.9 and 3.10. Although the definition of $r_{1}$ and $r_{2}$ is different from that of Uesugi et al. [19], it is clear that their results work well as the explicit conditions for Theorems 3.1-3.3. 


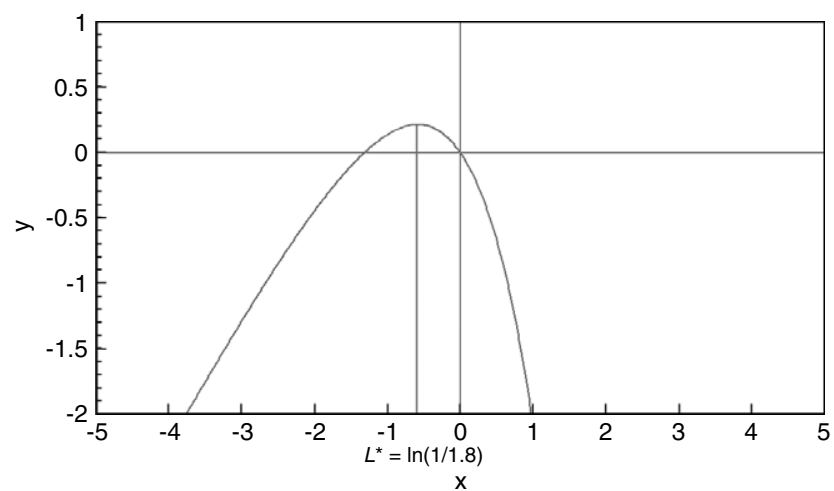

Fig. 1. Graph of $\varphi(x), r_{1}=1.8$.

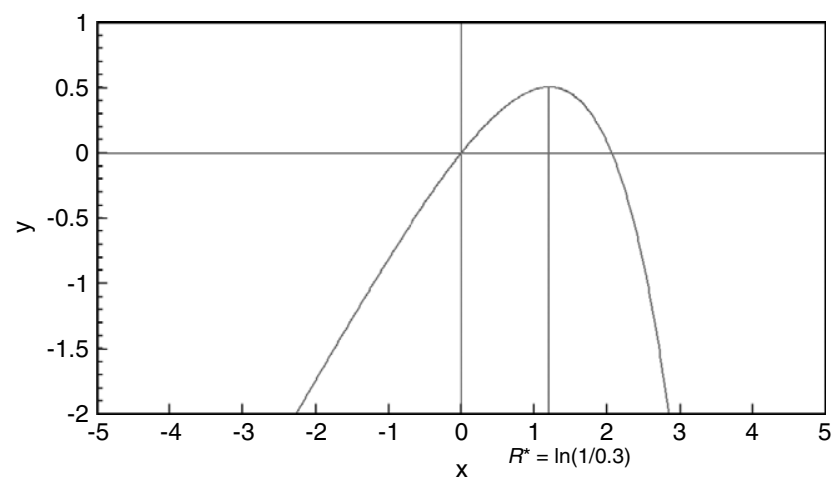

Fig. 2. Graph of $\varphi(x), r_{1}=0.3$.

Lemma 3.4 (See [19, Lemma 3.1]). Assume that

$$
0<r_{1}+r_{2} \leq 2 \text {. }
$$

Then,

$$
\left\{\begin{array}{l}
\tilde{\varphi}^{2}(L)>L \text { for any } L<0, \\
\tilde{\varphi}^{2}(R)<R \quad \text { for any } R>0,
\end{array}\right.
$$

and for (2.3) with $r_{2}=0, \lim _{n \rightarrow+\infty} x(n)=0$.

Lemma 3.4 implies that if $0<r_{1} \leq 2$ and $r_{2}=0$, then $\lim _{n \rightarrow+\infty} x(n)=0$.

Next, we introduce the following result.

Lemma 3.5. Assume that

$$
r_{1}>r_{2} \geq 0
$$

For $L \leq 0$, put

$$
H(L)=\varphi(L)-r_{2} f\left(R_{L}\right)-L,
$$

where

$$
R_{L}=\varphi\left(d_{L}\right)-r_{2} f(L)=d_{L} .
$$

Then,

$$
H(L)>0 \text { for any } L<0 .
$$

Proof. By (2.14), we have that $f\left(d_{L}\right)=-\frac{r_{2}}{r_{1}} f(L)$ for $L<0$. By (2.15), it follows that

$$
\begin{aligned}
H(L) & =-r_{1} f(L)+\frac{r_{2}^{2}}{r_{1}} f(L) \\
& =-\frac{1}{r_{1}} f(L)\left(r_{1}^{2}-r_{2}^{2}\right)>0 \text { for } L<0 .
\end{aligned}
$$

Hence, the proof is complete. 
From this lemma, we see that $r_{1}>r_{2} \geq 0$ ensures that (3.2) and (3.3) in Theorem 3.2 hold. Then, we obtain one of the main theorems, which extends the results given in $[9,20]$.

Theorem 3.6. Let $m \geq 1$. Suppose that $(\mathrm{H})$ holds. If

$$
r \leq \frac{1}{i+1}
$$

then for any solution $N(t)$ of (1.1), $\lim _{t \rightarrow+\infty} N(t)=N^{*}$ and the positive equilibrium $N^{*}$ is globally asymptotically stable.

Proof. By (2.6)-(2.8) and (3.6), we have that

$$
r_{1}>r_{2} \geq 0 \text { and } r_{1}+r_{2} \leq 1 \text {. }
$$

For the case $r_{2}=0$, by Lemma 3.4, we obtain the conclusion of this lemma. Let us consider the case $r_{2}>0$. From (3.7), $\varphi(x)$ attains a unique local maximum at $R^{*}=\ln \frac{1}{r_{1}}>0$. Then we see that

$$
-r_{1} f\left(R^{*}\right)+r_{2}=-r_{1} \exp \left(R^{*}\right)+r_{1}+r_{2} \leq 0
$$

from which it holds that $R^{*} \geq \varphi\left(R^{*}\right)+r_{2}$. By Lemma 3.5 and (1) in Theorem 3.2, we obtain the conclusion of this theorem.

Remark 3.7. For the case $i=0$, a result similar to the global stability is obtained by Muroya [9] and Wang et al. [20]. In particular, for the case $i=0$, in Muroya [9], the solution has contractivity.

Example 3.8. Consider the following logistic equation with piecewise constant arguments:

$$
\frac{\mathrm{d} N(t)}{\mathrm{d} t}=N(t) r\left(1-\sum_{j=0}^{4} b_{j} N([t-j])\right) .
$$

When $b_{0}>\sum_{j=1}^{4} b_{j}$ holds, the positive equilibrium $N^{*}$ is globally asymptotically stable if $r \leq 1$. For the case $\sum_{k=0}^{1} \sum_{j=0}^{1-k} b_{j}>\sum_{k=0}^{1} \sum_{j=2-k}^{4} b_{j}$, the positive equilibrium $N^{*}$ is globally asymptotically stable if $r \leq \frac{1}{2}$. Moreover, for the case $\sum_{k=0}^{2} \sum_{j=0}^{2-k} b_{j}>\sum_{k=0}^{2} \sum_{j=3-k}^{4} b_{j}$, the positive equilibrium $N^{*}$ is globally asymptotically stable if $r \leq \frac{1}{3}$.

On the other hand, (1.4) becomes $r \leq \frac{3}{2(1+4)}=0.3$ and, hence, the condition for the global stability of the equation is improved in all cases.

Hereafter, we restrict our attention to the case of $r_{1}+r_{2}>1$ and $r_{2}>0$.

Lemma 3.9 (See [19, Lemma 3.2]). Assume that

$$
r_{1}>r_{2}>0, \quad r_{1}>1, r_{1}+r_{2} \leq 2 \text { and } r_{1}+r_{2}-\frac{r_{2}}{r_{1}} \exp \left(r_{1}+r_{2}-1\right) \geq 0 \text {. }
$$

Then, $\varphi(x)$ attains a unique local maximum at $L^{*}=-\ln r_{1}<0$.

(1) For $L \leq 0$, put

$$
\left\{\begin{array}{l}
G_{1}(L)=\varphi(L)-r_{2} f\left(R_{L}^{*}\right)-L \\
\tilde{G}_{1}(L)=r_{1} f(L)+r_{2} f\left(R_{L}^{*}\right)
\end{array}\right.
$$

where

$$
R_{L}^{*}=\varphi\left(L^{*}\right)-r_{2} f(L) .
$$

Then, each of the following holds. (i) $\lim _{L \rightarrow-\infty} \tilde{G}_{1}(L) \leq 0$, (ii) $\tilde{G}_{1}\left(L^{*}\right)<0$, (iii) if $\tilde{G}_{1}^{\prime}(L)=0$ for some $L<L^{*}$, then $\tilde{G}_{1}(L)<0$. Hence, $\tilde{G}_{1}(L)<0$ and

$$
G_{1}(L)>0 \text { for any } L \leq L^{*}<0 .
$$

(2) For $L \leq 0$, put

$$
G_{2}(L)=\varphi\left(R_{L}^{*}\right)-r_{2} f\left(R_{L}^{*}\right)-L,
$$

where

$$
R_{L}^{*}=\varphi\left(L^{*}\right)-r_{2} f(L)
$$


Then, each of the following holds. (i) $\lim _{L \rightarrow-\infty} G_{2}(L)=+\infty$, (ii) $G_{2}\left(L^{*}\right)=\tilde{\varphi}^{2}\left(L^{*}\right)-L^{*}>0$, (iii) $G_{2}^{\prime}(L)<0$ for any $L<L^{*}$. Hence,

$$
G_{2}(L)>0 \text { for any } L \leq L^{*}<0 .
$$

(3) For $L \leq 0$, put

$$
G_{3}(L)=\varphi\left(\bar{R}_{L}\right)-r_{2} f\left(\bar{R}_{L}\right)-L,
$$

where

$$
\bar{R}_{L}=\varphi(L)-r_{2} f(L) .
$$

Then

$$
G_{3}(L)=\tilde{\varphi}^{2}(L)-L>0 \text { for any } L^{*} \leq L<0 .
$$

We note that $r_{1}>1$ ensures that $\varphi(x)$ is a unimodal function which has only one critical point at $L^{*}=-\ln r_{1}<0$. For the case $r_{1} \leq 1$, we introduce the following results.

Lemma 3.10 (See [19, Lemma 3.3]). Assume that

$$
1>r_{1}>r_{2}>0, \quad r_{1}+r_{2}>1 \text { and } r_{1}+r_{2}-\frac{r_{2}}{r_{1}} \exp \left(r_{1}+r_{2}-1\right) \geq 0 .
$$

Then, $\varphi(x)$ attains a unique local maximum at $R^{*}=-\ln r_{1}>0$.

(1) For $L \leq 0$, put

$$
\left\{\begin{array}{l}
G_{4}(L)=\varphi(L)-r_{2} f\left(R_{L}^{* *}\right)-L \\
\tilde{G}_{4}(L)=r_{1} f(L)+r_{2} f\left(R_{L}^{* *}\right)
\end{array}\right.
$$

where

$$
R_{L}^{* *}=\varphi\left(R^{*}\right)-r_{2} f(L) .
$$

Then, there exists a unique $\bar{L}<0$ such that $R^{*}=\varphi\left(R^{*}\right)-r_{2} f(\bar{L})$, and each of the following holds. (i) $\lim _{L \rightarrow-\infty} \tilde{G}_{4}(L) \leq$ 0 , (ii) $\tilde{G}_{4}(\bar{L})<0$, (iii) $\tilde{G}_{4}^{\prime}(\bar{L})>0$. Hence,

$$
G_{4}(L)>0 \text { for any } L \leq \bar{L}<0 .
$$

(2) For $L \leq 0$, put

$$
G_{5}(L)=\varphi\left(R_{L}^{* *}\right)-r_{2} f\left(R_{L}^{* *}\right)-L,
$$

where

$$
R_{L}^{* *}=\varphi\left(R^{*}\right)-r_{2} f(L) .
$$

Then, $\varphi\left(R_{L}^{* *}\right)>\varphi(L)$ and

$$
G_{5}(L)=\tilde{\varphi}\left(R_{L}^{* *}\right)-L>G_{4}(L)>0 \text { for any } L \leq \bar{L}<0 .
$$

Lemma 3.11 (See [19, Lemma 3.4]). Assume that

$$
r_{1}=1, \quad r_{2}>0 \text { and } 1+r_{2}-r_{2} \exp \left(r_{2}\right) \geq 0 .
$$

Then, $\varphi(x)$ attains a unique local maximum at $R^{*}=-\ln r_{1}=0$. There exists a unique $\bar{L}=0$ such that $R^{*}=\varphi\left(R^{*}\right)-r_{2} f(\bar{L})$.

(1) For (3.17) with $R^{*}=0$, it holds that

$$
G_{4}(L)>0 \text { for any } L<\bar{L}=0 .
$$

(2) For (3.19) with $R^{*}=0$, it holds that

$$
G_{5}(L)=\tilde{\varphi}\left(R_{L}^{* *}\right)-L>0 \text { for any } L<\bar{L}=0 .
$$

Now, we can prove Theorem 1.1. 
Proof of Theorem 1.1. From (1.9), (1.10) and (2.6)-(2.8), we have that

$$
r_{1}+r_{2} \leq 2 \text { and } r_{1}+r_{2}-\frac{r_{2}}{r_{1}} \exp \left(r_{1}+r_{2}-1\right) \geq 0 \text { for } r_{1}+r_{2}>1 \text {. }
$$

By Theorem 3.6, it is sufficient to give the proof for the case $r_{1}+r_{2}>1$. Now we easily see that (3.9), (3.16) and (3.21) are satisfied, respectively.

From (1) and (2) of Lemma 3.9, it holds that

$$
\min \left\{\varphi(L), \varphi\left(R_{L}^{*}\right)\right\}-r_{2} f\left(R_{L}^{*}\right)>L \text { for any } L \leq L^{*}<0,
$$

where

$$
R_{L}^{*}=\varphi\left(L^{*}\right)-r_{2} f(L) .
$$

Moreover, from (3) of Lemma 3.9, it holds that

$$
\varphi\left(\bar{R}_{L}\right)-r_{2} f\left(\bar{R}_{L}\right)>L \text { for any } L \in\left[L^{*}, 0\right),
$$

where

$$
\bar{R}_{L}=\varphi(L)-r_{2} f(L) .
$$

We note that $\min \left\{\varphi(L), \varphi\left(R_{L}\right)\right\}=\varphi\left(R_{L}\right)$ for $L^{*} \leq L<R_{L}$. Hence, (3.1) in Theorem 3.1 holds.

From (1), (2) of Lemmas 3.10 and 3.11, it holds that

$$
\min \left\{\varphi(L), \varphi\left(R_{L}^{* *}\right)\right\}-r_{2} f\left(R_{L}^{* *}\right)>L \text { for any } L<0,
$$

where

$$
R_{L}^{* *}=\varphi\left(R^{*}\right)-r_{2} f(L) .
$$

Hence, (3.1)-(3.4) in Theorems 3.2 and 3.3 hold.

By Theorems 3.1-3.3, we obtain the conclusion of this theorem. Hence, the proof is complete.

\section{Applications}

The object of this section is to introduce some examples and to compare our results with some previous results.

Consider the following logistic equation with piecewise constant arguments:

$$
\frac{\mathrm{d} N(t)}{\mathrm{d} t}=N(t) r\left(1-\sum_{j=0}^{2} b_{j} N([t-j])\right)
$$

(4.1) has the positive equilibrium $N^{*}=1 / \sum_{j=0}^{2} b_{j}$.

If $3 b_{0}+2 b_{1}+b_{2}>b_{1}+2 b_{2}$ then we choose $i=2$ such that $(\mathrm{H})$ holds. By Theorem 1.1, if

$$
r \leq \frac{2}{3}, \quad \text { and } \quad 3 r-\frac{b_{1}+2 b_{2}}{3 b_{0}+2 b_{1}+b_{2}} \exp (3 r-1) \geq 0 \text { for } r>\frac{1}{3},
$$

then the positive equilibrium $N^{*}$ is globally asymptotically stable.

If $2 b_{0}+b_{1}>b_{1}+2 b_{2}$ then we can choose $i=1$ such that $(\mathrm{H})$ holds. In this case, by Theorem 1.1 , if

$$
r \leq 1, \quad \text { and } \quad 2 r-\frac{b_{1}+2 b_{2}}{2 b_{0}+b_{1}} \exp (2 r-1) \geq 0 \quad \text { for } r>\frac{1}{2},
$$

then the positive equilibrium $N^{*}$ is globally asymptotically stable. On the other hand, (1.4) becomes $r \leq \frac{3}{2(2+1)}=0.5$.

Moreover, for the case $b_{0}>b_{1}+b_{2}$, we can choose $i=0$ such that $(\mathrm{H})$ holds. By Theorem 1.1 , if

$$
r \leq 2, \quad \text { and } \quad r-\frac{b_{1}+b_{2}}{b_{0}} \exp (r-1) \geq 0 \text { for } r>1,
$$

then the positive equilibrium $N^{*}$ is globally asymptotically stable. This is also shown by Theorem C.

Now, we fix $b_{1}=b_{2}=0.1$ for (4.1). By Corollary 1.2 , we see that if $b_{0} \geq \frac{1}{3}\left[\frac{0.3}{(2 / e)}-0.3\right] \simeq 0.03591 \ldots$ and $r \leq \frac{2}{3}$, then the positive equilibrium $N^{*}$ is globally asymptotically stable. If $b_{0} \geq \frac{1}{2}\left[\frac{0.3}{(2 / e)}-0.1\right] \simeq 0.15387 \ldots$ and $r \leq 1$, then the positive equilibrium $N^{*}$ is globally asymptotically stable. Moreover, if $b_{0} \geq \frac{0.2}{(2 / e)} \simeq 0.27182 \ldots$ and $r \leq 2$, then the positive equilibrium $N^{*}$ is globally asymptotically stable. 


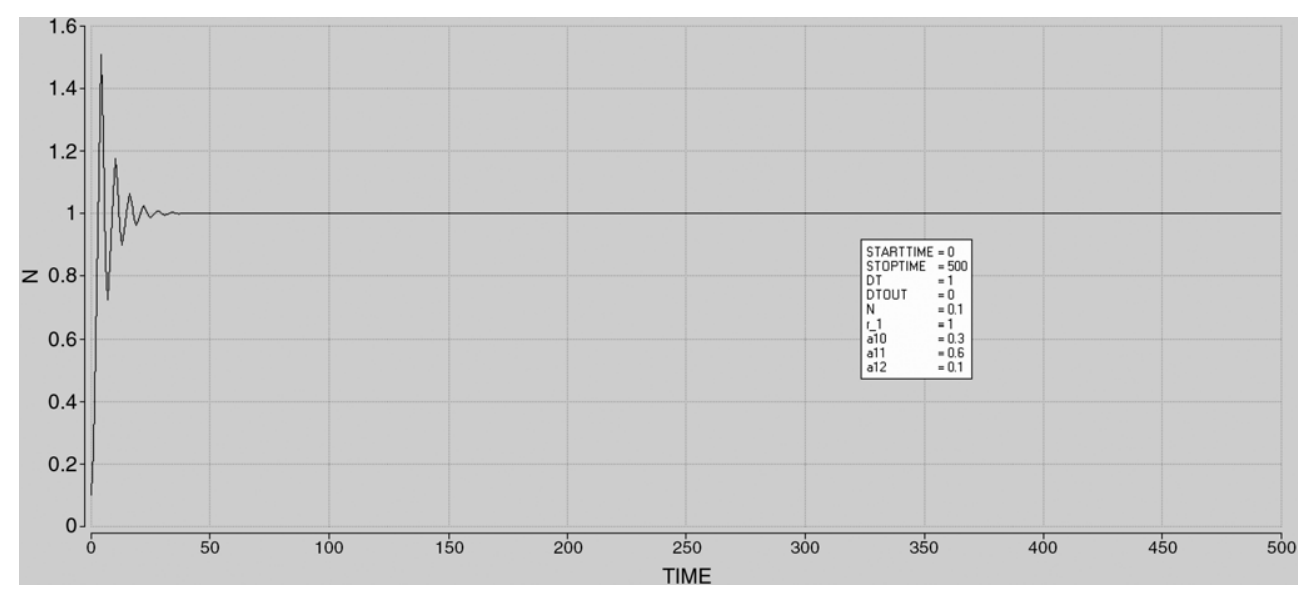

Fig. 3. (4.5) with $r=1$.

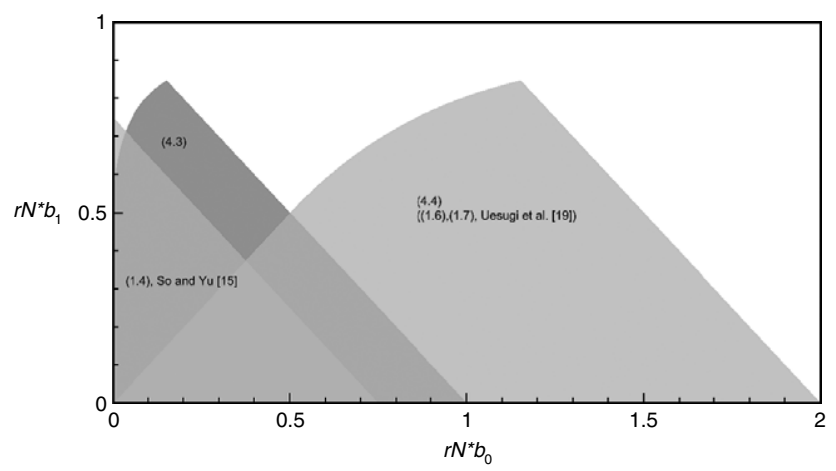

Fig. 4. Several global stability regions for (4.1) with $b_{2}=0$.

Example 4.1. The positive equilibrium $N^{*}=1$ of the following logistic equation with piecewise constant arguments:

$$
\frac{\mathrm{d} N(t)}{\mathrm{d} t}=N(t) r(1-0.3 N([t])-0.6 N([t-1])-0.1 N([t-2]))
$$

is globally asymptotically stable if $r \leq 1$.

In Fig. 3, we draw the orbit of the solution for (4.5) with $r=1$ and initial condition $\left(N_{0}, N_{-1}, N_{-2}\right)=(0.1,0.1,0.1)$.

Example 4.2. The positive equilibrium $N^{*}=\frac{1}{2}$ of the following logistic equation with piecewise constant arguments:

$$
\frac{\mathrm{d} N(t)}{\mathrm{d} t}=N(t) r(1-0.9 N([t])-1.1 N([t-2])),
$$

is globally asymptotically stable if $r \leq \frac{2}{3}$.

Fig. 4 illustrates our stability region and previous results for (4.1) with $b_{2}=0$. Moreover, in Fig. 5, some stability regions for (4.1) with $b_{1}=0$ are shown.

\section{Discussion}

In this paper, we generalize the approach in $[19,12]$ and establish a new sufficient condition for the global asymptotic stability of a logistic equation with piecewise constant arguments. Their results are given as one of the special cases of our result. Moreover, we improve the $3 / 2$ type stability condition for some suitable situations. The $3 / 2$ type condition ensures the global attractivity of the positive equilibrium of (1.1) for any set of the coefficients on the delay terms, however, our results state that the condition can be improved when taking account of the effect of delays. Our new approach is applicable to a wider class of delay difference equations and discrete systems. Such applications will be our future work. 


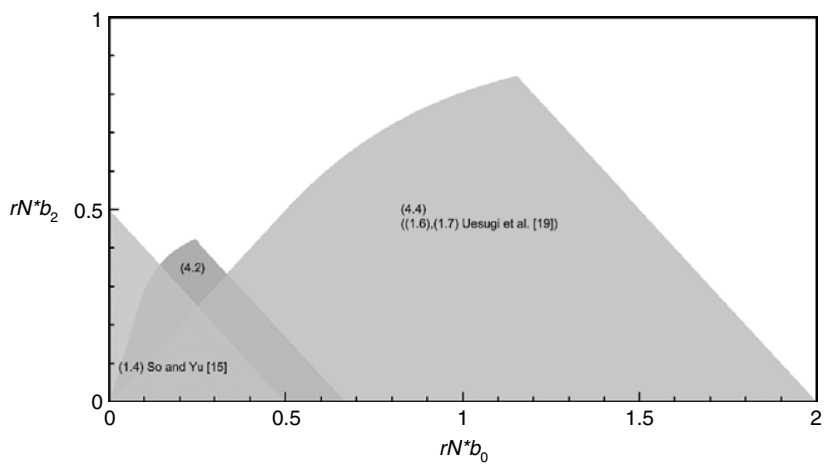

Fig. 5. Several global stability regions for (4.1) with $b_{1}=0$.

\section{Acknowledgements}

The author would like to thank Yuji Akimoto for continuous assistance and express his gratitude to Professor Yoshiaki Muroya for his valuable comments and numerous suggestions regarding this paper.

\section{References}

[1] K. Gopalsamy, P. Liu, Persistence and global stability in a population model, J. Math. Anal. Appl. 224 (1) (1998) 59-80.

[2] I. Györi, F. Hartung, On numerical approximation using differential equations with piecewise-constant arguments, Period. Math. Hungar. 56 (1) (2008) 55-69.

[3] H. Li, R. Yuan, An affirmative answer to Gopalsamy and Liu's conjecture in a population model, J. Math. Anal. Appl. 338 (2) (2008) $1152-1168$.

[4] L. Liao, J. Yu, L. Wang, Global attractivity in a logistic difference model with a feedback control, Comput. Math. Appl. 44 (10-11) (2002) $1403-1411$.

[5] P. Liu, K. Gopalsamy, Global stability and chaos in a population model with piecewise constant arguments, Appl. Math. Comput. 101 (1) (1999) 63-88.

[6] H. Matsunaga, T. Hara, S. Sakata, Global attractivity for a logistic equation with piecewise constant argument, NoDEA Nonlinear Differential Equations Appl. 8 (1) (2001) 45-52.

[7] H. Matsunaga, T. Hara, S. Sakata, Global attractivity for a nonlinear difference equation with variable delay, Comput. Math. Appl. 41 (5-6) (2001) 543-551.

[8] Y. Muroya, Persistence, contractivity and global stability in logistic equations with piecewise constant delays, J. Math. Anal. Appl. 270 (2) (2002) $602-635$.

[9] Y. Muroya, A sufficient condition on global stability in a logistic equation with piecewise constant arguments, Hokkaido Math. J. 32 (1) (2003) 75-83.

[10] Y. Muroya, Global attractivity for discrete models of nonautonomous logistic equations, Comput. Math. Appl. 53 (7) (2007) 1059-1073.

[11] Y. Muroya, E. Ishiwata, Stability for a class of difference equations, J. Comput. Appl. Math. 228 (2) (2009) 561-570.

[12] Y. Muroya, E. Ishiwata, N. Guglielmi, Global stability for nonlinear difference equations with variable coefficients, J. Math. Anal. Appl. 334 (1) (2007) $232-247$.

[13] Y. Muroya, Y. Kato, On Gopalsamy and Liu's conjecture for global stability in a population model, J. Comput. Appl. Math. 181 (1) (2005) $70-82$.

[14] Y. Nakata, M. Kuroda, Y. Muroya, Contractivity for nonautonomous logistic equation with piecewise constant delays, in: Proceedings of Mathematical Models in Engineering, Biology and Medicine, Conference on Boundary Value Problems, Santiago de Compostela, Spain, September 16-19, 2008.

[15] J.W.-H. So, J.S. Yu, Global stability in a logistic equation with piecewise constant arguments, Hokkaido Math. J. 24 (2) (1995) $269-286$.

[16] X. Tang, D. Cao, X. Zou, Global attractivity of positive periodic solution to periodic Lotka-Volterra competition systems with pure delay, J. Differential Equations 228 (2) (2006) 580-610.

[17] X. Tang, X. Zou, A $3 / 2$ stability result for a regulated logistic growth model, Discrete Contin. Dyn. Syst. Ser. B 2 (2) (2002) $265-278$.

[18] X.H. Tang, X. Zou, 3/2-type criteria for global attractivity of Lotka-Volterra competition system without instantaneous negative feedbacks, J. Differential Equations 186 (2) (2002) 420-439.

[19] K. Uesugi, Y. Muroya, E. Ishiwata, On the global attractivity for a logistic equation with piecewise constant arguments, J. Math. Anal. Appl. 294 (2) (2004) 560-580.

[20] W. Wendi, G. Mulone, F. Salemi, V. Salone, Global stability of discrete population models with time delays and fluctuating environment, J. Math. Anal. Appl. 264 (1) (2001) 147-167. 